\title{
Some Topological Indices and Their Polynomials of Graphene
}

\author{
H. L. PARASHIVAMURTHY ${ }^{1}$, M. R. RAJESH KANNA ${ }^{2 *}$ and R. JAGADEESH ${ }^{3}$ \\ 'BGS Institute of Technology, Adichunchanagiri University, B. G. Nagar-571448, \\ Nagamangala Taluk, Mandya District, India. \\ ${ }^{2}$ Department of Mathematics, Sri. D. DevarajaUrs Governement First Grade College, \\ Hunsur - 571 105, India. \\ ${ }^{3}$ Government Science College (Autonomous), Nrupathunga Road, \\ Bangalore -560001, Karnataka, India. \\ ${ }^{*}$ Corresponding author E-mail: mr.rajeshkanna@gmail.com
}

http://dx.doi.org/10.13005/ojc/350506

(Received: June 15, 2019; Accepted: September 01, 2019)

\section{ABSTRACT}

In this manuscript we have computed third Zagreb index, first Zagreb polynomial, second Zagreb polynomial, third Zagreb polynomial, hyper Zagreb polynomial, forgotten index, forgotten polynomial, symmetric division index and symmetric division polynomial of Graphene. These quantities are based on degrees of the vertices.

Mathematics Subject Classification: 05C12, 05C90

Keywords: Zagreb indices, Zagreb polynomials, Hyper Zagreb polynomial, Forgotten index, Forgotten polynomial, Symmetric division index, Symmetric division polynomial, Graphene.

\section{INTRODUCTION}

Graphene is a nanomaterial. Recently rajesh kanna and his students computed some of the topological indices of graphene ${ }^{1,2}$. In this article, we have computed third zagreb index, first zagreb polynomial, second zagreb polynomial, third zagreb polynomial, hyper zagreb index, forgotten index, symmetric division index. Also we have defined hyper zagreb polynomial, forgotten polynomial and symmetric division polynomial of graphene.

\section{Third zagreb index}

Fath-tabar introduced the third zagreb index, first, second and third zagreb polynomial in 2011 as follows ${ }^{3}$.

Definition 1.1: For a simple connected graph $\mathrm{G}$, the third zagreb index is defined as,

$Z G_{3}(G)=\sum_{e=u v \in E(G)}\left|d_{u}-d_{v}\right|$.

Definition 1. 2: The first, second and third zagreb polynomials for a simple connected graph $G$ is defined as,

$$
\begin{aligned}
& Z G_{1}(G, x)=\sum_{e=u v \in E(G)} x^{a_{u}+d_{v}} . \\
& Z G_{2}(G, x)=\sum_{e=u v \in E(G)} x^{d_{u} d_{v}} .
\end{aligned}
$$

This is an Open Access article licensed under a Creative Commons license: Attribution 4.0 International (CC- BY). Published by Oriental Scientific Publishing Company @ 2018 
$Z G_{3}(G, x)=\sum_{e=u v \in E(G)} x^{\left|a_{u}-a_{v}\right|}$

\section{Hyper zagreb index}

G. H. Shirdel et al., introduced a new distance-based zagreb indices of a graph $\mathrm{G}$ named hyper-zagreb index ${ }^{4}$.

Definition 1. 3: The hyper zagreb index is defined as,

$H Z(G)=\sum_{e=u v \in E(G)}\left(d_{u}+d_{v}\right)^{2}$

We define hyper zagreb polynomial as follows,

Definition 1. 4: The hyper zagreb polynomial is defined as,

$H Z(G, x)=\sum_{e=u \in E(G)} x^{\left(a_{x}+a_{v}\right)^{2}}$

\section{Forgotten index}

Definition 1. 5: The forgotten topological index is also a degree based topological index, denoted by $F(G)$ for simple graph $G$. It was encountered in ${ }^{5}$ and defined as,

$F(G)=\sum_{e=u v \in E(G)}\left[\left(d_{u}\right)^{2}+\left(d_{v}\right)^{2}\right]$.

Definition 1. 6: The forgotten polynomial for a graph $G$ is defined as,

$F(G)=\sum_{e-u v \in E(G)} x^{\left[\left(a_{u}\right)^{2}+\left(a_{v}\right)^{2}\right]}$

\section{Symmetric division index}

These topological indices are quite useful for determining total surface area and heat formation of some chemical compounds.

Definition 1. 7: Symmetric division index is defined as

$$
S D D(G)=\sum_{e=u v \in(G)}\left\{\frac{\min \left(d_{u}, d_{v}\right)}{\max \left(d_{u}, d_{v}\right)}+\frac{\max \left(d_{u}, d_{v}\right)}{\min \left(d_{u}, d_{v}\right)}\right\} .
$$

Further, we define symmetric division polynomial as follows

Definition 1. 8: The symmetric division polynomial is defined as

$S D D(G, x)=\sum_{e=u v \in(G)} x^{\left\{\frac{\min \left(d_{u}, d_{v}\right)}{\max \left(d_{u}, d_{v}\right)}+\frac{\max \left(d_{u}, d_{v}\right)}{\min \left(d_{u}, d_{v}\right)}\right\}}$

Main results

Theorem 2.1. The third zagreb index of Graphene, $Z G_{3}(G)= \begin{cases}4 s+2 t-4 & \text { if } t \neq 1 \\ 4(s-1) & \text { if } t=1\end{cases}$

Where ' $t$ ' is the number of rows of benzene rings and ' $s$ ' is the number of benzene rings in each row.

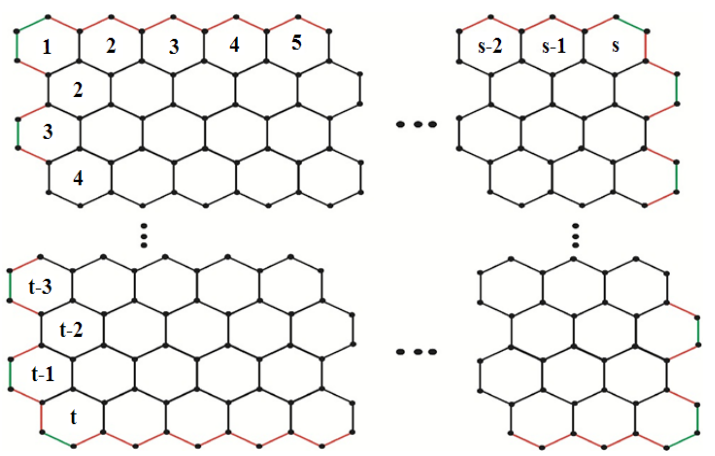

Fig. 1.

Proof: $2 d$ structure of graphene is as shown in the above Fig. 1. Assume that it contains 't' rows and ' $s$ ' benzene rings in every row. The edge connecting the vertices of degree di and $d_{i}$ is denoted by $m_{i, j}$. Let $I \mathrm{~m}_{\mathrm{i}, \mathrm{j}}$ denotes the number of edges of the type $m_{i, j} \cdot I n^{2}$ we can see that $\left|m_{2,2}\right|=(t+4),\left|m_{2,3}\right|=(4 s+$ $2 t-4)$ and $\left|m_{3,3}\right|=(3 t s-2 s-t-1)$.

Case1: If $\mathrm{t} \neq 1$, Consider, $Z G_{3}(G)=\sum_{e=u v \in E(G)}\left|d_{u}-d_{v}\right|$.

$=\left|m_{2,2}\right||2-2|+\left|m_{2,3}\right||2-3|+\left|m_{3,3}\right||3-3|$.

$=(t+4)(0)+(4 s+2 t-4)(1)+(3 t s-2 s-t-1)(0)$

$=4 \mathrm{~s}+2 \mathrm{t}-4$

$\therefore Z G_{3}(G)=4 s t+2 t-4$.

Case2: If $t=1, \ln ^{2}$ we can find $\left|m_{2,2}\right|=6,\left|m_{2,3}\right|=(4 s-4)$ and $\operatorname{lm}_{3,3} \mid=(\mathrm{s}-1)$ as in the following Fig. 2 below.

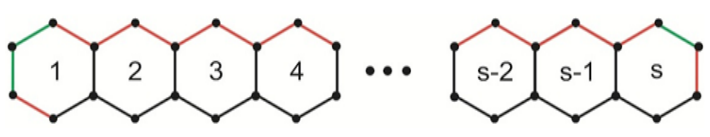

Fig. 2.

$$
\begin{aligned}
\text { Consider, } \quad z G_{3}(G)=\sum_{e=u \mathcal{u} \in E(G)}\left|d_{u}-d_{v}\right| . \\
=\left|m_{2,2}\right||2-2|+\left|m_{2,3}\right||2-3|+\left|m_{3,3}\right||3-3| \\
=6(0)+(4 s-4)(1)+(s-1)(0) \\
=4 s-4
\end{aligned}
$$

$\therefore Z G_{3}(G)=4(s-1)$, if $\mathrm{t}=1$.

Theorem 2. 2: First zagreb polynomial of Graphene,

$Z G_{1}(G, x)= \begin{cases}x^{4}\left[(3 t s-2 s-t-1) x^{2}+(4 s+2 t-4) x+(t+4)\right] & \text { if } t \neq 1 \\ x^{4}\left[(s-1) x^{2}+4(s-1) x+6\right] & \text { if } t=1\end{cases}$

Proof. Case1: If $t \neq 1$, Consider 


$$
\begin{aligned}
& Z G_{1}(G, x)=\sum_{e=u v \in E(G)} x^{d_{u}+d_{v}} . \\
& =\left|m_{2,2}\right| x^{(2+2)}+\left|m_{2,3}\right| x^{(2+3)}+\left|m_{3,3}\right| x^{(3+3)} \\
& =(t+4) x^{4}+(4 s+2 t-4) x^{5}+(3 t s-2 s-t-1) x^{6}+x^{4}[(3 t s-2 s-t- \\
& \left.1) x^{2}+(4 s+2 t-4) x+(t+4)\right] \\
& \quad Z G_{1}(G, x)=x^{4}\left[(3 t s-2 s-t-1) x^{2}+(4 s+2 t-4) x+(t+4)\right] i f t \neq 1 .
\end{aligned}
$$

Case 2: If $\mathrm{t}=1$,

$$
\begin{aligned}
& \boldsymbol{Z} G_{1}(G, x)=\sum_{e=u v \in E(G)} x^{a_{u}+d_{v}} . \\
& =\left|m_{2,2}\right| x^{(2+2)}+\left|m_{2,3}\right| x^{(2+3)}+\left|m_{3,3}\right| x^{(3+3)} \\
& =6 x^{4}+(4 s-4) x^{5}+(S-1) x^{6}
\end{aligned}
$$$$
Z G_{1}(G, x)=x^{4}\left[(s-1) x^{2}+4(s-1) x+6\right] \text {, if } t=1 \text {. }
$$

Theorem 2. 3 The second zagreb polynomial of graphene,

$Z C_{2}(G)= \begin{cases}x^{4}\left[(3 t s-2 s-t-1) x^{5}+(4 s+2 t-4) x^{2}+t+4\right] & \text { if } t \neq 1 \\ x^{4}\left[(s-1) x^{5}+4(s-1) x^{2}+6\right] & \text { if } t=1\end{cases}$

Proof: Case1: If $\mathrm{t} \neq 1$

$Z G_{2}(G, x)=\sum_{e=u v \in E(G)} x^{a_{u} a_{v}}$.

$=\left|m_{2,2}\right| x^{(2.2)}+\left|m_{2,3}\right| x^{(2.3)}+\left|m_{3,3}\right| x^{(3.3)}$

$=(t+4) x^{4}+(4 s+2 t-4) x^{6}+(3 t s-2 s-t-1) x^{9}$

$=x^{4}\left[(3 t s-2 s-t-1) x^{5}+(4 s+2 t-4) x^{2}+t+4\right]$

$$
Z G_{2}(G, x)=x^{4}\left[(3 t s-2 s-t-1) x^{5}+(4 s+2 t-4) x^{2}+t+4\right] \text { if } t \neq 1 .
$$

Case 2: If $\mathrm{t}=1$,

$$
\begin{aligned}
& \boldsymbol{Z} G_{\mathbf{Z}}(\boldsymbol{G}, \boldsymbol{x})=\sum_{\boldsymbol{e}=\boldsymbol{u v} \in_{E}(\boldsymbol{G})} \boldsymbol{x}^{\boldsymbol{d u}_{\boldsymbol{u}} \boldsymbol{d}_{\boldsymbol{v}} .} \\
& =\left|m_{2,2}\right| x^{(2.2)}+\left|m_{2,3}\right| x^{(2.3)}+\left|m_{3,3}\right| x^{(3.3)} \\
& =6 x^{4}+(4 s-4) x^{6}+(S-1) x^{9} \\
& Z G_{1}(G, x)=x^{4}\left[(s-1) x^{5}+4(s-1) x^{2}+6\right], \text { if } t=1 .
\end{aligned}
$$

Theorem 2. 4 Third zagreb polynomial of graphene,

$$
Z G_{3}(G)= \begin{cases}{[(4 s+2 t-4) x+3 t s-2 s+3]} & \text { if } t \neq 1 \\ {[4(s-1) x+s+5]} & \text { if } t=1\end{cases}
$$

Proof: Case1: If $t \neq 1$

$$
z G_{3}(G, x)=\sum_{e=u v \in E(G)} x^{\left|d_{u}-d_{v}\right|} \text {. }
$$

$$
\begin{aligned}
& =\left|m_{2,2}\right| x^{|2-2|}+\left.\left|m_{2,3}\right|\right|^{|2-3|}+\left.\left|m_{3,3}\right|\right|^{|3-3|} \\
& =(t+4) x^{0}+(4 s+2 t-4) x^{1}+(3 t s-2 s-t-1) x^{0} \\
& =[(4 s+2 t-4) x+3 t s-2 s-t-1+t+4] \\
& \quad 2 G_{3}(G, x)=[(4 s+2 t-4) x+3 t s-2 s-t-1+t+4] \text { if } t \neq 1 .
\end{aligned}
$$

Case 2: If $\mathrm{t}=1$

$$
\begin{aligned}
& Z G_{3}(G, x)=\sum_{e=u v \in E(G)} x^{\left|d_{u}-a_{v}\right|} . \\
& =\left|m_{2,2}\right| x^{|2-2|}+\left|m_{2,3}\right| x^{|2-3|}+\left|m_{3,3}\right| x^{|3-3|} \\
& =6 x^{0}+(4 s-4) x^{1}+(s-1) x^{0} \\
& =4(s-1) x+s-1+6 \\
& Z G_{1}(G, x)=[4(s-1) x+s+5], \text { if } t=1 .
\end{aligned}
$$

Theorem 2. 5 Hyper zagreb polynomial of graphene,

$H Z(G, x)=\left\{\begin{array}{cc}{\left[x^{16}(3 t s-2 s-t-1) x^{20}+(4 s+2 t-4) x^{9}+t+4\right]} & \text { if } t \neq 1 \\ x^{16}\left[(s-1) x^{20}+4(s-1) x^{9}+6\right] & \text { if } t=1\end{array}\right.$

Proof: Case1: If $t \neq 1$

$$
\begin{aligned}
& H Z(G, x)=\sum_{e=u v \in E(G)} x^{\left(a_{u}+a_{v}\right)^{2}} \\
& =\left|m_{2,2}\right| x^{|2+2|^{2}}+\left|m_{2,3}\right| x^{|2+3|^{2}}+\left|m_{3,3}\right| x^{|3+3|^{2}} \\
& =(t+4) x^{16}+(4 s+2 t-4) x^{25}+(3 t s-2 s-t-1) x^{36} \\
& =x^{16}\left[(3 t s-2 s-1) x^{20}+(4 s+2 t-4) x^{9}+t+4\right]
\end{aligned}
$$

$\mathrm{HZ}(\mathrm{G}, \mathrm{X})=x^{16}\left[(3 t s-2 s-1) x^{20}+(4 s+2 t-4) x^{9}+t+4\right]$, for $t=1$

\section{Case 2: If $\mathrm{t}=1$}

$H Z(G, x)=\sum_{e=u v \in E(G)} x^{\left(a_{u}+a_{v}\right)^{2}}$.

$$
\begin{aligned}
& =\left|m_{2,2}\right| \mathrm{x}^{(2+2)^{2}}+\left|m_{2,3}\right| x^{|2+3|^{2}}+\left|m_{3,3}\right| x^{|3+3|^{2}} \\
& =6 x^{16}+(4 s-4) x^{25}+(s-1) x^{36} \\
& =x^{16}\left[(s-1) x^{20}+4(s-1) x^{9}+6\right]
\end{aligned}
$$

$H Z(G, x)=x^{16}\left[(s-1) x^{20}+4(s-1) x^{9}+6\right]$, for $t=1$.

Theorem 2.6 Forgotten topological index of graphene,

$$
F(G)= \begin{cases}{[2(27 t s+8 s+8 t-19)]} & \text { if } t \neq 1 \\ {[2(35 s-11]} & \text { if } t=1\end{cases}
$$

Proof: Case 1:If $t=1$ 
$F(G)=\sum_{e=u v \in E(G)}\left[\left(d_{u}\right)^{2}+\left(d_{v}\right)^{2}\right.$

$=\left|m_{2,2}\right|\left(2^{2}+2^{2}\right)+\left|m_{2,3}\right|\left(2^{2}+3^{2}\right)+\left|m_{3,3}\right|\left(3^{2}+3^{2}\right)$

$=(\mathrm{t}+4)(8)+(4 \mathrm{~s}+2 \mathrm{t}-4)(13)+(3 \mathrm{ts}-2 \mathrm{~s}-\mathrm{t}-1)(18)$

$=8 \mathrm{t}+32+52 \mathrm{~s}+26 \mathrm{t}-52+54 \mathrm{ts}-36 \mathrm{~s}-18 \mathrm{t}-18$

$=54 \mathrm{ts}+16 \mathrm{~s}+16 \mathrm{t}-38$

$=2(27 \mathrm{ts}+8 \mathrm{~s}+8 \mathrm{t}-19)$

$\mathrm{F}(\mathrm{G})=2(27 \mathrm{ts}+8 \mathrm{~s}+8 \mathrm{t}-19)$, for $\mathrm{t}=1$.

Case 2: If $\mathrm{t}=1$

$F(G)=\sum_{e=u v \in E(G)}\left[\left(d_{u}\right)^{2}+\left(d_{v}\right)^{2}\right.$

$=\left|m_{2,2}\right|\left(2^{2}+2^{2}\right)+\left|m_{2,3}\right|\left(2^{2}+3^{2}\right)+\left|m_{3,3}\right|\left(3^{2}+3^{2}\right)$

$=6(8)+(4 \mathrm{~s}-4)(13)+(\mathrm{s}-1)(18)$

$=48+52 \mathrm{~s}-52+18 \mathrm{~s}-18$

$=70 \mathrm{~s}-22$

$=2(35 \mathrm{~s}-11)$

$\mathrm{F}(\mathrm{G})=2(35 \mathrm{~s}-11), 40=1$

Theorem 2. 7 Forgotten polynomial of graphene $F(G, x)=\left\{\begin{array}{cc}{\left[x^{8}(3 t s-2 s-t-1) x^{10}+(4 s+2 t-4) x^{5}+t+4\right]} & \text { if } t \neq 1 \\ x^{8}\left[(s-1) x^{10}+4(s-1) x^{5}+6\right] & \text { if } t=1\end{array}\right.$

Proof: case1: If $\mathrm{t} \neq 1$ is

$$
\begin{aligned}
F(G, x)= & \sum_{e=u v \in E(G)} x\left(\left(a_{u}\right)^{2}+\left(a_{v}\right)^{2}\right. \\
& =\left|m_{2,2}\right| x^{\left(2^{2}+2^{2}\right)}+\left.\left|m_{2,3}\right|\right|^{\left(2^{2}+3^{2}\right)}+\left|m_{3,3}\right| x^{\left(3^{2}+3^{2}\right)}
\end{aligned}
$$

$=(t+4) x^{8}+(4 s+2 t-4) x^{13}+(3 t s-2 s-t-1) x^{18}$

$=x^{8}\left[(3 t s-2 s-t-1) x^{10}+(4 s+2 t-4) x^{5}+t+4\right]$

$F(G, x)=x^{8}\left[(3 t s-2 s-t-1) x^{10}+(4 s+2 t-4) x^{5}+t+4\right)$, fort $=1$

Case 2: If $\mathrm{t}=1$

$F(G, x)=\sum_{e=u v \in E(G)} x^{\left(\left(d_{u}\right)^{2}+\left(d_{v}\right)^{2}\right.}$

$=\left|m_{2,2}\right| x^{\left(2^{2}+2^{2}\right)}+\left|m_{2,3}\right| x^{\left(2^{2}+3^{2}\right)}+\left|m_{3,3}\right| x^{\left(3^{2}+3^{2}\right)}$

$=6 x^{8}+(4 s-4) x^{13}+(S-1) x^{18}$

$=x^{8}\left[(s-1) x^{10}+4(s-1) x^{5}+6\right]$

$F(G, x)=x^{8}\left[(s-1) x^{10}+4(s-1) x^{5}+6\right]$, for $t=1$.
Theorem 2. 8 The symmetric division index of graphene is

$\operatorname{SDD}(\mathrm{G})=\left\{\begin{array}{cc}6 t s+(4.33333) t+(4.066667) s-2.066667 & \text { if } t \neq 1 \\ (10.66667) s+1.33333) & \text { if } t=1\end{array}\right.$

Proof : Case 1: for $t \neq 1$

$$
\begin{aligned}
& \operatorname{sDD}(G)=\sum_{G=w \in \in(G)}\left\{\frac{\min \left(d_{w}, d_{v}\right)}{\max \left(d_{w}, d_{v}\right)}+\frac{\max \left(d_{w}, d_{v}\right)}{\min \left(d_{w} d_{v}\right)}\right\} . \\
& =\left|m_{2,2}\right|\left\{\frac{\min (2,2)}{\max (2,2)}+\frac{\max (2,2)}{\min (2,2)}\right\}+\left|m_{2,3}\right|\left\{\frac{\min (2,3)}{\max (2,3)}+\frac{\max (2,3)}{\min (2,3)}\right\} \\
& +\left|m_{3,3}\right|\left\{\frac{\min (3,3)}{\max (3,3)}+\frac{\max (3,3)}{\min (3,3)}\right\}
\end{aligned}
$$

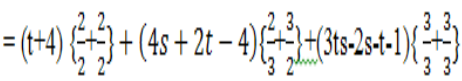

$\left.=(t+4)\{2\}+(4 s+2 t-4)\left\{\frac{13}{6}\right\}+(3 t s-2 s-t-1)\right)\{2\}$

$=6 \operatorname{ts}+(4.333333) \mathrm{t}+(4.666667) \mathrm{s} \cdot 2.66667$

$\operatorname{SDD}(\mathrm{G})=6 \operatorname{ts}+(4.333333) \mathrm{t}+(4.666667) \mathrm{s}-2.66667$, for $\mathrm{t} \neq 1$.

Case 2: If $t=1$ is

$$
\begin{gathered}
\operatorname{SDD}(G)=\sum_{\varepsilon=u v \in(G)}\left\{\frac{\min \left(d_{w^{\prime}} d_{v}\right)}{\max \left(d_{w^{\prime}} d_{v}\right)}+\frac{\max \left(d_{w^{\prime}} d_{v}\right)}{\min \left(d_{w^{\prime}} d_{v}\right)}\right\} . \\
\left.\left.=\left|m_{2,2}\right| \frac{\min (2,2)}{\max (2,2)}+\frac{\max (2,2)}{\min (2,2)}\right\}+\left|m_{2,3}\right| \frac{\min (2,3)}{\max (2,3)}+\frac{\max (2,3)}{\min (2,3)}\right\} \\
\left.+\left|m_{3,3}\right| \frac{\{\min (3,3)}{\max (3,3)}+\frac{\max (3,3)}{\min (3,3)}\right\}
\end{gathered}
$$

$=6\left\{\frac{2}{2}+\frac{2}{2}\right\}+(4 s-4)\left\{\frac{2}{3}+\frac{2}{2}\right\}+(s-1)\left\{\frac{3}{3}+\frac{3}{3}\right\}$

$=6\{2\}=(4 s-4)\left\{\frac{13}{6}\right\}+(s-1)\{2\}$

$=\left\{\frac{32 s+4}{3}\right\}$

$=(10.66667) s+1.33333$

$\operatorname{SDD}(\mathrm{G})=(17.33333) \mathrm{s}+34.66667$, for $\mathrm{t}=1$.

Theorem 2. 9 The symmetric division polynomial of graphene

$\mathrm{SDD}(\mathrm{G}, \mathrm{x})=\left\{\begin{array}{r}(4 s+2 t-4) x^{\frac{13}{6}}(3 t s-2 s+3) x^{2} \text { if } t \neq 1 \\ 4(s-1) x^{\frac{13}{6}}+(s+5) x^{2} \text { if } t=1\end{array}\right.$

Proof: Case 1: If $\mathrm{t} \neq 1$,

$S D D(G)=\sum_{e=u v \in(G)} x^{\left\{\frac{\min \left(d_{u}, d_{v}\right)}{\max \left(d_{u}, d_{v}\right)}+\frac{\max \left(d_{u}, d_{v}\right)}{\min \left(d_{u}, d_{v}\right.}\right\}}$

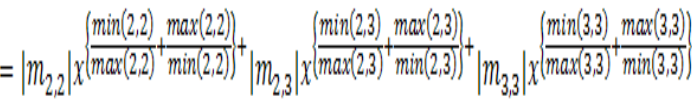




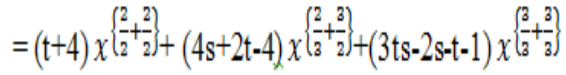

$$
\begin{aligned}
& =(t+4) x^{2}+(4 s+2 t-4) x^{\frac{13}{6}}+(3 t s-2 s-t-1) x^{2} \\
& =(4 s+2 t-4) x^{\frac{13}{6}}+(3 t s-2 s+3) x^{2} \\
& \mathrm{SDD}(\mathrm{G}, \mathrm{x})=(4 s+2 t-4) x^{\frac{13}{6}}+(3 t s-2 s+3) x^{2}, \text { fort } \neq 1 \text {. }
\end{aligned}
$$

Case 2: For $\mathrm{t}=1$,

$$
\begin{aligned}
& S D D(G, x)=\sum_{e=u v \in(G)} x^{\left\{\frac{\min \left(d_{u}, d_{v}\right)}{\max \left(d_{u}, d_{v}\right)}+\frac{\max \left(d_{u}, d_{v}\right)}{\min \left(d_{u}, d_{v}\right)}\right\}}
\end{aligned}
$$

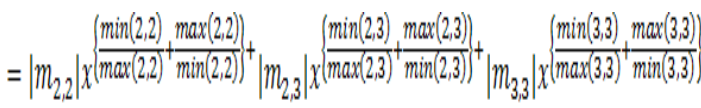

$$
\begin{aligned}
& =6 x^{\left\{\frac{2}{2}+\frac{2}{2}\right\}}+(4 \mathrm{~s}-4) x^{\left\{\frac{2}{3}+\frac{3}{2}\right\}}+(\mathrm{s}-1) x^{\left\{\frac{3}{3}+\frac{3}{3}\right\}} \\
& =6 x^{2}+(4 s-4) x^{\frac{13}{6}}+(s-1) x^{2} \\
& =(4 s-4) x^{\frac{13}{6}}+(s+5) x^{2}
\end{aligned}
$$

$\mathrm{SDD}(\mathrm{G}, \mathrm{x})=(4 s-4) x^{\frac{13}{6}}+(s+5) x^{2}$ for $\mathrm{t}=1$.

\section{CONCLUSION}

In this article we have computed general formula for third zagreb index, hyper zagreb polynomial, first zagreb polynomial, second zagreb polynomial third zagreb polynomial, forgotten index, forgotten polynomial, symmetric division index and symmetric division polynomial of graphene.

\section{ACKNOWLEDGMENT}

This research did not receive any specific grant from funding agencies in the public, commercial, or not-for-profit sectors.

\section{Conflict of interests}

The authors declare that there is no conflict of interests regarding the publication of this article.

\section{Authors contributions}

All the authors worked together for the preparation of manuscript and all of us take the full responsibility for the content of the article, however first author typed the article and all of us read and approved the final manuscript.

\section{REFERENCES}

1. Jagadeesh R. M.; R. Rajesh Kanna and Indumathi R. Some results on topological indices of Graphene, nanomaterials and nanotechnology., 2016, 6, 1-6.

2. G. Shridhara.; M. R Rajesh Kanna and R. S Indumathi. Computation of Topological Indices of Graphene., Journal of Nanomaterials., 2015, 8.

3. Ali Astanesh-asl and G. H Fath-tabar, computing first and third zagreb polynomials of Certain product of graphs, Iranian Jounal of Mathematical Chemistry., 2011, 2-2, 73-78.

4. G. H Shirdel, H. Rezapour and amsayadi. Hyper zagreb index of graph operations, Iranian Journal of Mathematical Chemistry., 2013, 4(2), 213-230.

5. B. Furtula and Gutman. I. A forgotten topological index, Journal of Mathematical Chemistry., 2015, 53, 213-220. 